\title{
IN ROAD TRAFFIC ACCIDENTS, ARE THE PATTERN, SEVERITY AND OUTCOME OF DRIVERS' INJURIES AFFECTED BY MARIJUANA ABUSE?
}

\author{
Amira A. Wahdan ${ }^{a}$ and Amal S.A.F. Hafez ${ }^{a}$ \\ ${ }^{a}$ Forensic Medicine and Clinical Toxicology Department, Faculty of Medicine, Tanta \\ University, Tanta, Egypt.
}

Corresponding author: Amira A. Wahdan Forensic Medicine and Clinical Toxicology Department $-6^{\text {th }}$ floor Faculty of Medicine - Tanta University - Medical Campus - El-Gash Street - Tanta - El-Gharbia Governorate Egypt.

Email: amira.wahdan@med.tanta.edu.eg

Telephone number: +201147824441

Submit Date 2021-02-22

Revise Date 2021-04-24

Accept Date 2021-04-30

\begin{abstract}
Introduction: Road traffic accidents (RTAs) represent a leading contributor to global disease burden. Drivers involved in some RTAs were found to be under the influence of different drugs. Marijuana effects on driving risks are not thoroughly understood. The study aimed to compare the pattern, severity and clinical outcome of injuries between marijuanasmoking drivers and non-drugged drivers in RTAs. Patients and Methods: This prospective, comparative study was carried out on 78 drivers admitted to emergency department, Tanta University Emergency Hospital during the period from the start of January to the end of December 2018. All drivers were subjected to history taking, clinical examination, assessment of injury severity score (ISS) and revised trauma score (RTS) and detection of tetrahydrocannabinol (THC) in urine. Results: Thirty-six drivers tested positive for THC with a median level of $314.6 \mathrm{ng} / \mathrm{ml}$. There was lack of significant association between marijuana smoking and the site of injuries. The marijuana-positive group had a significantly higher frequency of skull fractures, ruptured spleen, intraperitoneal hemorrhage, and abrasions on body surface when compared to the marijuana-negative group. The median ISS was nonsignificantly higher, and the mortality was significantly higher in the marijuana-positive group. The THC level correlated significantly and strongly with both ISS and RTS. A significantly higher median THC level was detected in non-survivors compared to survivors. Conclusion: It could be concluded that Marijuana smoking is associated with increased mortality rate in victims of RTAs. There are strong correlations between THC level and both ISS and RTS, suggesting a dose-dependent effect of marijuana smoking.
\end{abstract}

Keywords: Accidents, Marijuana, Smoking, Tetrahydrocannabinol, Score

\section{INTRODUCTION}

Road traffic accidents (RTAs) are considered among the leading causes of morbidity and mortality, both on national and world-wide scales. In 2017, RTAs were reported to result in injuries to approximately 54 million victims worldwide, out of whom approximately 1 million died (James et al., 2020). The Global Status Report on Road Safety (World Health Organization, 2018) stated that crash injuries occupied the eighth rank as the cause of death worldwide, regardless of age. The same report shows that RTAsrelated fatalities in Egypt were 9287 people in 2016, representing a rate of 9.7 per 100 000 population.

Drivers involved in some RTAs were found to be under the influence of different drugs. Common drugs which were detected in drivers after RTAs included marijuana, alcohol, and benzodiazepines. The hazards 
associated with driving under the influence of alcohol are well-documented. Nevertheless, the impact of marijuana intake on driving and the associated risks are not precisely defined nor explained. This factor in addition to being the most widely consumed illicit substance worldwide make it a growing global concern (Andrews et al., 2015; Das et al., 2020).

According to Hartman Huestis (2013), cannabis smoking impacts the nervous system, causing some effects such as diminished reflexes, weaving and lanechanging, as well as cognitive dysfunctions. These cannabis-induced effects are associated with higher risk of the development of RTAs. People driving under the influence of cannabis demonstrated aggressive driving habits and risk-taking behavior that can impair driving skills (Ramaekers et al., 2006; BergeronPaquette, 2014). Furthermore, cannabis use was found to affect, in a doserelated manner, the ability of the driver to control the motor vehicle, resulting in mistakes that reflect the impairment of sensory perception, motor control and cognitive function (Martin et al., 2017; Das et al., 2020).

Subsequently, both RTAs and driving under cannabis influence represent growing global public health concerns. Factors related to human behavior, vehicles and road are contributing to the causality of RTAs (Mir et al., 2012). Concerning human behavior, cannabis is the second frequent substance distinguished in victims of fatal RTAs in Australia, UK, United States and many European countries (Drummer et al., 2012; HartmanHuestis, 2013; Martin et al., 2017; Das et al., 2020). However, the risks induced by cannabis are underestimated by cannabis abusers, being mistakenly regarded as the "least risky illicit drug" (Arterberry et al., 2013).

In Egypt, more than 20 thousand crashes occur annually, most of which by drug-influenced truck drivers (Obada,
2009). Therefore, the current study aimed to compare the pattern, severity, and clinical outcome of injuries between marijuana smoking drivers and non-drugged drivers in RTAs.

\section{PATIENTS \& METHODS}

Study design and settings

This prospective, comparative study was carried out on drivers admitted to emergency department, Tanta University Emergency Hospital. after obtaining approval from the research ethical committee of Tanta Faculty of Medicine (approval code: 34075 ).

\section{Ethical considerations}

Detailed information concerning the aim and methods of the study was provided prior to acquiring an informed written consent from patients or their relatives (if the patient was incompetent and cannot be involved in the consent process). Patients' confidentiality was preserved as each patient was assigned a specific code number and all data collection sheets were kept anonymous.

\section{Subjects}

This study included 78 drivers suffering from recent RTAs admitted to emergency department, Tanta University Emergency Hospital from the $1^{\text {st }}$ of January 2018 to the $31^{\text {st }}$ of December 2018.

Inclusion criteria:

Drivers (from both genders) admitted to emergency department, Tanta University Emergency Hospital suffering from recent RTAs causing injuries in different parts of their bodies were included.

\section{Exclusion criteria:}

The following drivers were excluded from the study: a) drivers who were recurring for check-ups; b) those who abused substances other than marijuana; c) those who abused multiple substances; and d) drivers with history of current diseases that might precipitate RTAs (epilepsy, diabetes mellitus, coronary heart disease, hypertension, liver diseases and problems of binocular vision).

\section{Methods of the study}

Cases were divided into two groups: 
non-drugged drivers and marijuana smoking drivers.

All were subjected to:

1. History taking with emphasis on personal history (age, gender, residence and special habits), past history (including any medical disease other than those in the exclusion criteria, operations and history of previous admissions for traumatic injury) and pre-hospital care (if any intervention took place before reaching hospital).

2. Clinical examination: including vital signs, consciousness level by Glasgow Coma Scale (GCS) and neurological examination

3. Examination of injuries: Assessment of injuries, recording and categorizing them according to their anatomical site.

4. Injury Severity Score (ISS): is a scoring system which is commonly used in injury victims, particularly those sustaining multiple traumata. The calculation of ISS entails the estimation of Abbreviated Injury Scale (AIS), which is an anatomical score dividing the body into 6 areas: head and neck, face, chest, abdomen, pelvis and extremities and skin. Each injury on the AIS gets a score of 1-6 (1 is minor and 6 is fatal). To calculate the ISS, every injury is scored using the AIS, then the highest scores in the three most injured body regions are identified, squared, and summed up. The minimum ISS score is 1 and the maximum is 75 (Nayeem et al., 1992; Kumaraguru et al., 2015).

5. Revised Trauma Score (RTS): The RTS is a physiological scoring system that is based on GCS, systolic blood pressure and the respiratory rate. To determine the RTS, these three parameters are assigned codes ranging from 4 (normal) to 0 (table 1). The following equation is used to calculate RTS

$\mathrm{RTS}=0.9368 \mathrm{GCS}+0.7326 \mathrm{SBP}+$ $0.2908 \mathrm{RR}$, where GCS, SBP, and RR are replaced by the corresponding coding values from table 1 . The RTS ranges from 0 to 8 (Nayeem et al., 1992; Petridou Antonopoulos, 2017).

\begin{tabular}{|l|l|l|l|}
\hline $\begin{array}{l}\text { Table (1): Values for Revised Trauma } \\
\text { Score (Nayeem et al., 1992): }\end{array}$ \\
\hline $\begin{array}{l}\text { Glasgow } \\
\begin{array}{l}\text { Coma } \\
\text { Scale }\end{array}\end{array}$ & $\begin{array}{l}\text { Systolic } \\
\text { blood } \\
\text { pressure }\end{array}$ & $\begin{array}{l}\text { Respiratory } \\
\text { rate }\end{array}$ & $\begin{array}{l}\text { Coded } \\
\text { value }\end{array}$ \\
\hline $13-15$ & $>89$ & $10-29$ & 4 \\
\hline $9-12$ & $76-89$ & $>29$ & 3 \\
\hline $6-8$ & $50-75$ & $6-9$ & 2 \\
\hline $4-5$ & $1-49$ & $1-5$ & 1 \\
\hline 3 & 0 & 0 & 0 \\
\hline
\end{tabular}

6. Laboratory investigations: (for qualitative and quantitative detection of cannabinoids in urine): Five $\mathrm{ml}$ urine sample was obtained from each patient on admission before receiving any treatment. Blood-containing and turbid samples were excluded. Patients who were comatose and/or unable to void urine were catheterized. Samples were collected, coded and dated in dry clean labeled containers. Each sample was subjected to rapid qualitative screening by Acro Rapid Test Diagnostic (Acro Biotech, Inc, California, USA). It is an enzyme immunoassay multi-drug rapid test panel kits for qualitative screening of 7 drugs; opiates, tramadol, cocaine, cannabinoids, benzodiazepines, barbiturates and amphetamines. Positive urine samples were subjected to cannabinoids immunoassay using the semi-quantitative, immunochemical automated analyzer the Abbott AxSYM ${ }^{\circledR}$ (Abbott Laboratories, Illinois, United States). This step was done to detect tetrahydrocannabinol (THC) level with cut off value $50 \mathrm{ng} / \mathrm{ml}$ (JosephRonald, 2006).

Statistical analysis

Statistical analysis was performed using SPSS software for Windows, version 26 (IBM Corp., Armonk, N.Y., USA). The Shapiro-Wilk test was carried out to determine distribution of continuous numerical data. Normally distributed data were summarized as mean \pm standard deviation (SD); comparisons between marijuana-positive and negative groups were carried out using independent samples T-test. Abnormally distributed data were summarized using the median and 
interquartile range (IQR: expressed as 25th - 75th percentiles); groups were compared using Mann-Whitney test. Correlations between numerical and/or ordinal variables were tested using Spearman's rank-order correlation. Categorical data were summarized as frequencies and associations between groups and categorical variables were assessed using Pearson's Chi square test, Fisher's exact test, or Fisher-FreemanHalton exact test. Statistical significance was adopted at a p-value $\leq 0.05$ for interpretation of statistical tests.

\section{RESULTS}

The present study was carried out on 78 drivers admitted with recent road traffic injury. Analysis of cannabinoid levels in urine showed that $42(53.8 \%)$ drivers tested negative, whereas the remaining $36(46.2 \%)$ tested positive. The median level of THC in positive cases was $314.6 \mathrm{ng} / \mathrm{ml}$ (range 56.8 - $1470.9 \mathrm{ng} / \mathrm{ml}$ ).

Table (2) summarizes the sociodemographic data of the studied drivers. Their age ranged from 18 to 63 years old, with a median of 27 years. Nearly half the drivers belonged to the age group (18 - <28), while those 48 years or older had the least frequency (9\%). Moreover, a higher percentage of marijuana-positive drivers belonged to the age group (18 $<28$ ), but no significant association ( $\mathrm{p}=$ 0.051) was detected between marijuana abuse and age groups. Men outnumbered women (91\% vs. 9\%, respectively). Drivers living in urban areas constituted $55.1 \%$, while those from rural areas constituted $44.9 \%$. Most drivers (71.8\%) were smokers, whereas drug abuse was admitted by only $23.1 \%$ of patients. All drivers in the positive group were smokers compared to $47.6 \%$ in the marijuana-negative group $(\mathrm{p}<0.001)$; and the prevalence of drug abusers was significantly higher in the positive group ( $38.9 \%$ vs. $9.5 \%, \mathrm{p}=0.002)$.

The vital signs, level of consciousness and clinical neurological examination were normal in most drivers. Tachypnea was recorded in a significantly higher percentage of marijuana-positive cases
(52.8\% vs. $26.2 \%, \mathrm{p}=0.016)($ Table 3$)$

Association of marijuana smoking with the distribution and type of injuries is displayed in table (4). No significant association was detected between marijuana smoking and the site of injuries, though a higher percentage of this group had injuries of abdominal and pelvic contents $(22.2 \%$ vs. $7.1 \%, \mathrm{p}=0.056)$. Compared to the marijuana-negative group, the marijuana-positive group had a higher percentage of skull fractures $(25 \%$ vs. $7.1 \%$, $\mathrm{p}=0.029)$, ruptured spleen $(16.7 \%$ vs. $2.4 \%, \quad \mathrm{p}=0.044)$, intraperitoneal hemorrhage $(22.2 \%$ vs $4.8 \%, p=0.038)$ and abrasions on body surface $(77.8 \%$ vs. $42.9 \%, \mathrm{p}=0.002)$. On the other hand, significantly higher percentage of marijuana-negative cases had contused wounds on the body surface $(59.5 \%$ vs. $36.1 \%, \mathrm{p}=0.039$ ).

The ISS and RTS were compared between the two groups in table (5). The median ISS was non-significantly higher in the marijuana-positive group ( 12 vs. $10, p=$ 0.434). Marijuana administration was significantly associated with higher mortality (36.1\% vs. $9.5 \%, \mathrm{p}=0.005)$.

The correlation between THC level, in marijuana-positive drivers, and each of ISS and RTS was significant and presented in fig. (1) and (2). The THC level positively and strongly correlated with ISS ( $\mathrm{rs}=0.717$, $\mathrm{p}<0.001)$. A negative, strong correlation was detected between THC levels and RTS ( $\mathrm{rs}=0.840, \mathrm{p}<0.001)$. Table (6) compares the THC level between surviving and nonsurviving marijuana-positive drivers, showing that non-survivors had a significantly higher median THC level than that of survivors $(934.8$ vs. $120.4 \mathrm{ng} / \mathrm{ml}$, respectively, $\mathrm{p}<0.001)$.

Table (7) summarizes the injuries found in non-surviving drivers, the ISS and the most probable, apparent causes of death. Death was caused by severe head injury in the four deceased marijuana-negative drivers. In marijuana-positive drivers, death was due to severe head injuries in 9 cases and to abdominal injuries in 4 cases. 
Table (2): Sociodemographic data in the studied victims of trauma (total $n=78$ )

\begin{tabular}{|c|c|c|c|c|c|c|}
\hline & & $\begin{array}{c}\text { All } \\
\text { participants } \\
(n=78)\end{array}$ & $\begin{array}{c}\text { Marijuana } \\
\text { negative } \\
\text { drivers } \\
(n=42)\end{array}$ & $\begin{array}{c}\text { Marijuana } \\
\text { positive } \\
\text { drivers } \\
(\mathbf{n}=\mathbf{3 6})\end{array}$ & $\begin{array}{c}\text { Test } \\
\text { statistic }\end{array}$ & $\mathbf{p}$ \\
\hline \multicolumn{2}{|c|}{$\begin{array}{c}\text { Age (years) } \\
\text { Median [IQR] } \\
\text { (range) }\end{array}$} & $\begin{array}{c}27.0[21.0- \\
38.0](18.0- \\
63.0)\end{array}$ & $\begin{array}{c}29.5[22.0- \\
44.0](18.0- \\
63.0)\end{array}$ & $\begin{array}{c}25.5[20.5- \\
31.5](18.0- \\
46.0)\end{array}$ & $1.728^{\mathrm{a}}$ & 0.084 \\
\hline \multirow{4}{*}{$\begin{array}{c}\text { Age } \\
\text { groups }\end{array}$} & $18-<28$ & $40(51.3 \%)$ & $18(42.9 \%)$ & $22(61.1 \%)$ & \multirow{4}{*}{$7.653^{b}$} & \multirow{4}{*}{0.051} \\
\hline & $28-<38$ & $17(21.8 \%)$ & $9(21.4 \%)$ & $8(22.2 \%)$ & & \\
\hline & $38-<48$ & $14(17.9 \%)$ & $8(19.0 \%)$ & $6(16.7 \%)$ & & \\
\hline & 48 or above & $7(9.0 \%)$ & $7(16.7 \%)$ & $0(0.0 \%)$ & & \\
\hline \multirow{2}{*}{ Sex } & Male & $71(91.0 \%)$ & $36(85.7 \%)$ & $35(97.2 \%)$ & \multirow{2}{*}{$\mathrm{FE}$} & \multirow{2}{*}{0.116} \\
\hline & Female & $7(9.0 \%)$ & $6(14.3 \%)$ & $1(2.8 \%)$ & & \\
\hline \multirow{2}{*}{ Residence } & Urban & $43(55.1 \%)$ & $21(50.0 \%)$ & $22(61.1 \%)$ & \multirow{2}{*}{$0.967^{\mathrm{c}}$} & \multirow{2}{*}{0.325} \\
\hline & Rural & $35(44.9 \%)$ & $21(50.0 \%)$ & $14(38.9 \%)$ & & \\
\hline \multirow{2}{*}{ Smoking } & No & $22(28.2 \%)$ & $22(52.4 \%)$ & $0(0.0 \%)$ & \multirow{2}{*}{$26.265^{c}$} & \multirow{2}{*}{$<0.001 *$} \\
\hline & Yes & $56(71.8 \%)$ & $20(47.6 \%)$ & $36(100.0 \%)$ & & \\
\hline \multirow{2}{*}{$\begin{array}{l}\text { Drug } \\
\text { abuse }\end{array}$} & No & $60(76.9 \%)$ & $38(90.5 \%)$ & $22(61.1 \%)$ & \multirow{2}{*}{$9.416^{\mathrm{c}}$} & \multirow{2}{*}{$0.002 *$} \\
\hline & Yes & $18(23.1 \%)$ & $4(9.5 \%)$ & $14(38.9 \%)$ & & \\
\hline
\end{tabular}

a : Mann-Whitney test; ${ }^{\text {b}}$ : Fisher-Freeman-Halton exact test; ${ }^{\text {c: }}$ Pearson's Chi square test for independence; FE: Fisher's exact test; IQR: interquartile range; * significant at $p$ $\leq \mathbf{0 . 0 5}$.

Table (3): Circumstances of trauma and clinical findings on admission in the studied victims of trauma (total $\mathrm{n}=78$ )

\begin{tabular}{|c|c|c|c|c|c|c|}
\hline & & $\begin{array}{l}\text { All participants } \\
(n=78)\end{array}$ & $\begin{array}{c}\text { Marijuana } \\
\text { negative } \\
\text { drivers } \\
(\mathrm{n}=\mathbf{4 2}) \\
\end{array}$ & $\begin{array}{c}\text { Marijuana } \\
\text { positive } \\
\text { drivers } \\
(\mathbf{n}=\mathbf{3 6}) \\
\end{array}$ & $\begin{array}{c}\text { Test } \\
\text { statistic }\end{array}$ & $\mathbf{p}$ \\
\hline \multirow[t]{2}{*}{ Time } & Daytime & $18(23.1 \%)$ & $5(11.9 \%)$ & $13(36.1 \%)$ & \multirow[t]{2}{*}{$6.399^{\mathrm{a}}$} & 0.011 \\
\hline & Night & $60(76.9 \%)$ & $37(88.1 \%)$ & $23(63.9 \%)$ & & $*$ \\
\hline \multirow{4}{*}{$\begin{array}{c}\text { Blood } \\
\text { pressure } \\
(\mathrm{mmHg})\end{array}$} & $\begin{array}{l}\text { Systolic Mean } \\
\pm \text { SD (range) }\end{array}$ & $\begin{array}{l}110.1 \pm 14.4 \\
(60.0-130.0)\end{array}$ & $\begin{array}{l}110.0 \pm 14.9 \\
(60.0-130.0)\end{array}$ & $\begin{array}{c}110.3 \pm 14.0 \\
(70.0-130.0)\end{array}$ & $0.084^{b}$ & 0.933 \\
\hline & $\begin{array}{l}\text { Diastolic Mean } \\
\pm \text { SD (range) }\end{array}$ & $\begin{array}{c}68.4 \pm 9.0 \\
(40.0-90.0)\end{array}$ & $\begin{array}{c}68.1 \pm 10.7 \\
(40.0-90.0)\end{array}$ & $\begin{array}{c}68.8 \pm 9.0 \\
(50.0-90.0) \\
\end{array}$ & $0.289^{b}$ & 0.773 \\
\hline & Normal & $68(87.2 \%)$ & $37(88.1 \%)$ & $31(86.1 \%)$ & \multirow[t]{2}{*}{$\mathrm{FE}$} & \multirow[t]{2}{*}{1.000} \\
\hline & Hypote & $10(1$ & $5(11.9 \%)$ & $5(13.9 \%)$ & & \\
\hline \multirow[t]{3}{*}{$\begin{array}{c}\text { Pulse } \\
\text { (beat/minute }\end{array}$} & $\begin{array}{l}\text { Mean } \pm \text { SD } \\
\text { (range) }\end{array}$ & $\begin{array}{c}85.6 \pm 17.2 \\
(60.0-130.0)\end{array}$ & $\begin{array}{c}87.2 \pm 18.0 \\
(60.0-130.0)\end{array}$ & $\begin{array}{c}83.7 \pm 16.2 \\
(60.0-123.0)\end{array}$ & $0.903^{b}$ & 0.370 \\
\hline & Normal & $64(82.1 \%)$ & $35(83.3 \%)$ & $29(80.6 \%)$ & \multirow[t]{2}{*}{$0.102^{a}$} & 0.775 \\
\hline & Tachycardia & $14(17.9 \%)$ & $7(16.7 \%)$ & $7(19.4 \%)$ & & \\
\hline \multirow[t]{2}{*}{$\begin{array}{c}\text { Temperature } \\
{ }^{\circ} \mathrm{C}\end{array}$} & $\begin{array}{l}\text { Mean } \pm \text { SD } \\
\text { (range) }\end{array}$ & $\begin{array}{c}36.9 \pm 0.3 \\
(36.5-37.7) \\
\end{array}$ & $\begin{array}{c}36.9 \pm 0.3 \\
(36.5-37.2) \\
\end{array}$ & $\begin{array}{c}36.8 \pm 0.4 \\
(36.5-37.7) \\
\end{array}$ & $1.624^{b}$ & 0.109 \\
\hline & Normal & $78(100.0 \%)$ & $42(100.0 \%)$ & $36(100.0 \%)$ & N/A & N/A \\
\hline \multirow{3}{*}{$\begin{array}{l}\text { Respiratory } \\
\text { rate (cycle/ } \\
\text { minute) }\end{array}$} & $\begin{array}{l}\text { Mean } \pm \text { SD } \\
\text { (range) }\end{array}$ & $\begin{array}{c}19.6 \pm 4.2 \\
(12.0-34.0)\end{array}$ & $\begin{array}{c}18.2 \pm 2.9 \\
(12.0-23.0)\end{array}$ & $\begin{array}{c}21.2 \pm 4.9 \\
(16.0-34.0)\end{array}$ & $3.198^{b}$ & $\begin{array}{c}0.002 \\
*\end{array}$ \\
\hline & Normal & $48(61.5 \%)$ & $31(73.8 \%)$ & $17(47.2 \%)$ & \multirow[t]{2}{*}{$5.789^{\mathrm{a}}$} & 0.016 \\
\hline & Tachypnea & $30(38.5 \%)$ & $11(26.2 \%)$ & $19(52.8 \%)$ & & \\
\hline
\end{tabular}




\begin{tabular}{|c|c|c|c|c|c|c|}
\hline GCS & $13-15$ & $57(73.1 \%)$ & $34(81.0 \%)$ & $23(63.9 \%)$ & $2.973^{\mathrm{c}}$ & 0.212 \\
\hline & $9-12$ & $9(11.5 \%)$ & $3(7.1 \%)$ & $6(16.7 \%)$ & & \\
\hline & $3-8$ & $12(15.4 \%)$ & $5(11.9 \%)$ & $7(19.4 \%)$ & & \\
\hline Clinical & Normal & $60(76.9 \%)$ & $35(83.3 \%)$ & $25(69.4 \%)$ & $2.106^{\mathrm{a}}$ & 0.147 \\
\hline neurological & Headache & $7(9.0 \%)$ & $2(4.8 \%)$ & $5(13.9 \%)$ & FE & 0.239 \\
\hline exam & Vertigo & $1(1.3 \%)$ & $0(0.0 \%)$ & $1(2.8 \%)$ & FE & 0.462 \\
\hline & Agitation & $3(3.8 \%)$ & $1(2.4 \%)$ & $2(5.6 \%)$ & $\mathrm{FE}$ & 0.593 \\
\hline & Convulsions & $2(2.6 \%)$ & $1(2.4 \%)$ & $1(2.8 \%)$ & FE & 1.000 \\
\hline & Hypotonia & $6(7.7 \%)$ & $3(7.1 \%)$ & $3(8.3 \%)$ & FE & 1.000 \\
\hline & $\begin{array}{l}\text { Diminished } \\
\text { tendon reflex }\end{array}$ & $3(3.8 \%)$ & $3(7.1 \%)$ & $0(0.0 \%)$ & FE & 0.245 \\
\hline
\end{tabular}

a: Pearson's Chi square test for independence; ${ }^{\text {b: }}$ Independent samples T-test; ${ }^{\text {c: }}$ FisherFreeman-Halton exact test; FE: Fisher's exact test; N/A: non-applicable; SD: standard deviation; * significant at $\mathbf{p} \leq \mathbf{0 . 0 5}$.

Table (4): Association of marijuana smoking with the distribution and type of injuries in the studied victims of trauma (total $n=78$ )

\begin{tabular}{|c|c|c|c|c|c|c|}
\hline & & $\begin{array}{c}\text { All } \\
\text { participants } \\
(\mathbf{n}=78)\end{array}$ & $\begin{array}{c}\text { Marijuana } \\
\text { negative } \\
\text { drivers } \\
(\mathrm{n}=42) \\
\end{array}$ & \begin{tabular}{|c|}
$\begin{array}{c}\text { Marijuana } \\
\text { positive } \\
\text { drivers } \\
(\mathbf{n}=36)\end{array}$ \\
\end{tabular} & $\begin{array}{c}\text { Test } \\
\text { statistic }\end{array}$ & $\mathbf{P}$ \\
\hline \multirow{4}{*}{$\begin{array}{l}\text { Head \& } \\
\text { Neck }\end{array}$} & Total cases & $24(30.8 \%)$ & $13(31.0 \%)$ & $11(30.6 \%)$ & $0.001^{\mathrm{a}}$ & 0.970 \\
\hline & \begin{tabular}{|l|} 
Skull fracture \\
\end{tabular} & $10(12.8 \%)$ & $3(7.1 \%)$ & $9(25.0 \%)$ & $4.748^{\mathrm{a}}$ & $0.029 *$ \\
\hline & Meningeal hemorrhage & $11(14.1 \%)$ & $3(7.1 \%)$ & $8(22.2 \%)$ & $3.639^{\mathrm{a}}$ & 0.056 \\
\hline & $\begin{array}{c}\text { Concussion/Cerebral } \\
\text { injury }\end{array}$ & $10(12.8 \%)$ & $8(19.0 \%)$ & $2(5.6 \%)$ & $\mathrm{FE}$ & 0.097 \\
\hline \multirow{3}{*}{ Face } & Total cases & $2(2.6 \%)$ & $2(4.8 \%)$ & $0(0.0 \%)$ & $\mathrm{FE}$ & 1.000 \\
\hline & Facial laceration & $1(1.3 \%)$ & $1(2.4 \%)$ & $0(0.0 \%)$ & $\mathrm{FE}$ & 1.000 \\
\hline & $\begin{array}{l}\text { Fracture body of } \\
\text { mandible }\end{array}$ & $1(1.3 \%)$ & $1(2.4 \%)$ & $0(0.0 \%)$ & $\mathrm{FE}$ & 1.000 \\
\hline \multirow{3}{*}{ Chest } & Total cases & $13(16.7 \%)$ & $6(14.3 \%)$ & $7(19.4 \%)$ & $0.371^{\mathrm{a}}$ & 0.542 \\
\hline & Rib fracture & $11(14.1 \%)$ & $5(11.9 \%)$ & $6(16.7 \%)$ & $0.363^{\mathrm{a}}$ & 0.547 \\
\hline & Clavicle fracture & $3(3.8 \%)$ & $1(2.4 \%)$ & $2(5.6 \%)$ & $\mathrm{FE}$ & 0.593 \\
\hline \multirow{4}{*}{$\begin{array}{l}\text { Abdominal } \\
\text { \& pelvis }\end{array}$} & Total cases & $11(14.1 \%)$ & $3(7.1 \%)$ & $8(22.2 \%)$ & $3.639^{\mathrm{a}}$ & 0.056 \\
\hline & Rupture spleen & $7(9.0 \%)$ & $1(2.4 \%)$ & $6(16.7 \%)$ & FE & $0.044 *$ \\
\hline & $\begin{array}{c}\text { Intraperitoneal } \\
\text { hemorrhage }\end{array}$ & $10(12.8 \%)$ & $2(4.8 \%)$ & $8(22.2 \%)$ & $\mathrm{FE}$ & $0.038 *$ \\
\hline & $\begin{array}{l}\text { Retroperitoneal } \\
\text { hematoma }\end{array}$ & $1(1.3 \%)$ & $1(2.4 \%)$ & $0(0.0 \%)$ & $\mathrm{FE}$ & 1.000 \\
\hline \multirow{3}{*}{$\begin{array}{c}\text { Bony pelvis } \\
\& \\
\text { Extremities }\end{array}$} & Total cases & $32(41.0 \%)$ & $20(47.6 \%)$ & $12(33.3 \%)$ & $1.635^{\mathrm{a}}$ & 0.201 \\
\hline & Fracture pelvis & $4(5.1 \%)$ & $2(4.8 \%)$ & $2(5.6 \%)$ & $\mathrm{FE}$ & 1.000 \\
\hline & Fracture extremities & $30(38.5 \%)$ & $19(45.2 \%)$ & $11(30.6 \%)$ & $1.766^{\mathrm{a}}$ & 0.184 \\
\hline \multirow{5}{*}{$\begin{array}{l}\text { Body } \\
\text { surface }\end{array}$} & Total cases & $78(100.0 \%)$ & $42(100.0 \%)$ & $36(100.0 \%)$ & N/A & N/A \\
\hline & Abrasions & $46(59.0 \%)$ & $18(42.9 \%)$ & $28(77.8 \%)$ & $9.770^{\mathrm{a}}$ & $0.002 *$ \\
\hline & Bruises & $59(75.6 \%)$ & $33(78.6 \%)$ & $26(72.2 \%)$ & $0.424^{\mathrm{a}}$ & 0.515 \\
\hline & Contused wound & $38(48.7 \%)$ & $25(59.5 \%)$ & $13(36.1 \%)$ & $4.253^{\mathrm{a}}$ & $0.039 *$ \\
\hline & Cut wound & $19(24.4 \%)$ & $8(19.0 \%)$ & $11(30.6 \%)$ & $1.393^{\mathrm{a}}$ & 0.238 \\
\hline
\end{tabular}

a: Pearson's Chi square test for independence; FE: Fisher's exact test; N/A: nonapplicable; * significant at $\mathrm{p} \leq \mathbf{0 . 0 5}$ 
Table (5): Comparison between marijuana negative and positive drivers as regards the injury severity score, revised trauma score and outcome

\begin{tabular}{|c|c|c|c|c|c|c|}
\hline \multicolumn{2}{|c|}{} & Total & $\begin{array}{c}\text { Marijuana } \\
\text { negative drivers } \\
(\mathbf{n}=\mathbf{4 2})\end{array}$ & $\begin{array}{c}\text { Marijuana } \\
\text { positive } \\
\text { drivers } \\
(\mathbf{n = 3 6 )}\end{array}$ & $\begin{array}{c}\text { Test } \\
\text { statistic }\end{array}$ & p \\
\hline $\begin{array}{c}\text { Injury } \\
\text { Severity Score }\end{array}$ & $\begin{array}{c}\text { Median [IQR] } \\
(\text { range) }\end{array}$ & $\begin{array}{c}10[5-20] \\
(1-57)\end{array}$ & $\begin{array}{c}10[5-17] \\
(2-34)\end{array}$ & $\begin{array}{c}12[4-29] \\
(1-57)\end{array}$ & $0.782^{\mathrm{a}}$ & 0.434 \\
\hline \multirow{2}{*}{ RTS } & $\begin{array}{c}\text { Median [IQR] } \\
(\text { range) }\end{array}$ & $\begin{array}{c}8[7-8] \\
(3-8)\end{array}$ & $\begin{array}{c}8[8-8] \\
(4-8)\end{array}$ & $\begin{array}{c}8[7-8] \\
(3-8)\end{array}$ & $1.410^{\mathrm{a}}$ & 0.159 \\
\hline \multirow{2}{*}{ Outcome } & Survivors & $\begin{array}{c}61 \\
(78.2 \%)\end{array}$ & $38(90.5 \%)$ & $23(63.9 \%)$ & \multirow{2}{*}{$8.039^{\mathrm{b}}$} & $0.005^{*}$ \\
\cline { 2 - 8 } & Non-survivors & $\begin{array}{c}17 \\
(21.8 \%)\end{array}$ & $4(9.5 \%)$ & $13(36.1 \%)$ & & \\
\hline
\end{tabular}

a: Mann-Whitney test; ${ }^{\text {b}}$ : Pearson's Chi square test for independence; IQR: interquartile range; * significant at $\mathrm{p} \leq \mathbf{0 . 0 5}$.

Table (6): Comparison between surviving and non-surviving marijuana-positive drivers (total $\mathrm{n}=36$ ) regarding tetrahydrocannabinol (THC) level

\begin{tabular}{|c|c|c|c|c|c|}
\hline & $\begin{array}{c}\text { Total } \\
(\mathbf{n}=\mathbf{3 6}) \\
\end{array}$ & $\begin{array}{c}\text { Survivors } \\
(\mathrm{n}=\mathbf{2 3})\end{array}$ & $\begin{array}{c}\text { Non-survivors } \\
(\mathbf{n}=\mathbf{1 3})\end{array}$ & $\begin{array}{c}\text { Mann- } \\
\text { Whitney test }\end{array}$ & $\mathbf{p}$ \\
\hline $\begin{array}{c}\text { THC level } \\
\text { (ng/ml) } \\
\text { Median [IQR] } \\
\text { (range) }\end{array}$ & $\begin{array}{c}314.6[84.3- \\
841.4] \\
(56.8-1470.9)\end{array}$ & $\begin{array}{c}120.4[70.8- \\
305.6](56.8 \\
-420.4)\end{array}$ & $\begin{array}{c}934.8[807.9- \\
1169.1](405.8- \\
1470.9)\end{array}$ & 4.891 & $<0.001 *$ \\
\hline
\end{tabular}

IQR: interquartile range; * significant at $\mathrm{p} \leq \mathbf{0 . 0 5}$

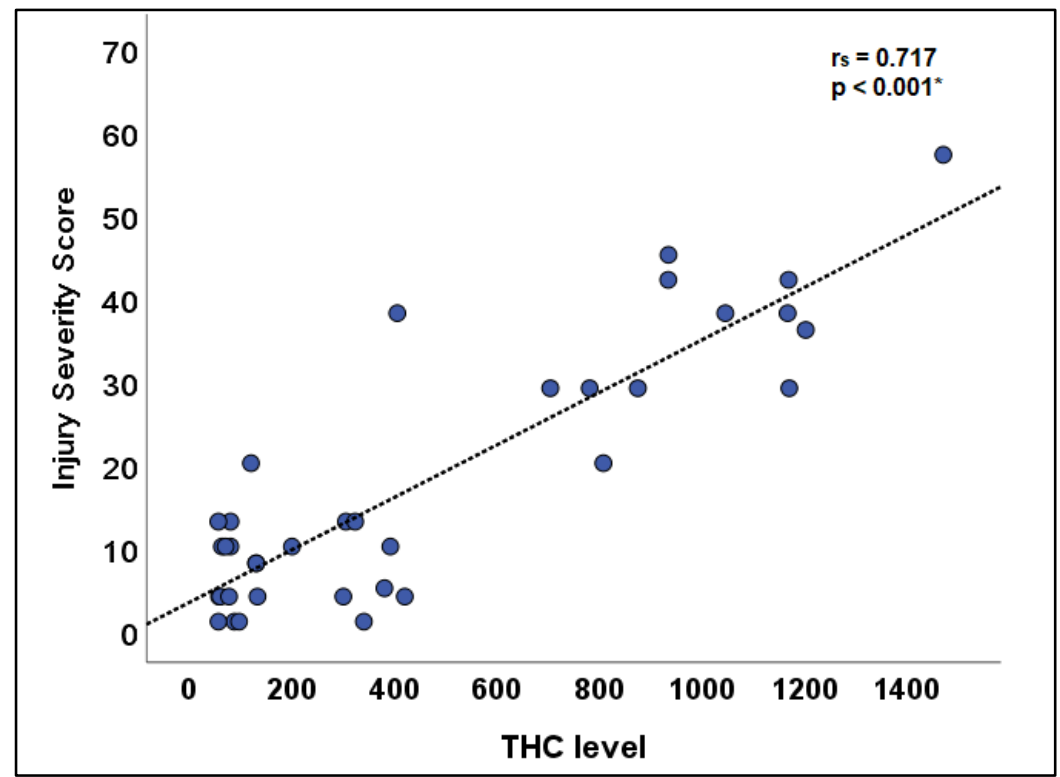

$\mathbf{r}_{\mathrm{s}}$ : Correlation coefficient; * significant at $\mathbf{p} \leq \mathbf{0 . 0 5}$.

Figure (1): Scatterplot showing correlation between tetrahydrocannabinol (THC) level and ISS in marijuana positive cases (total $n=36$ ). 


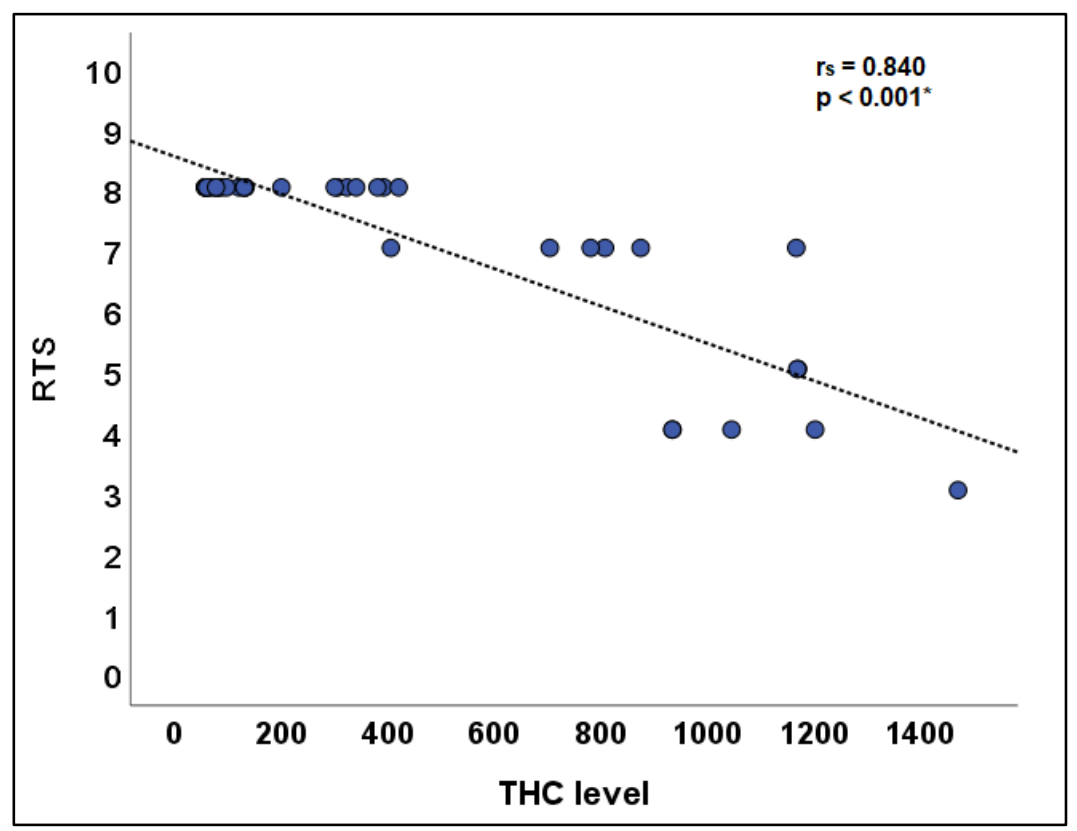

$\mathrm{r}_{\mathrm{s}}$ : Correlation coefficient; * significant at $\mathbf{p} \leq \mathbf{0 . 0 5}$.

Figure (2): Scatterplot showing correlation between tetrahydrocannabinol (THC) level and RTS in marijuana positive cases (total $\mathrm{n}=36$ ).

Table (7): Injuries, injury severity score and apparent causes of death in non-surviving drivers (total $\mathrm{n}=17$ )

\begin{tabular}{|c|c|c|c|c|}
\hline Case & Group & Injuries & ISS & Apparent cause of death \\
\hline 1 & $\begin{array}{l}\text { Marijuana- } \\
\text { negative driver }\end{array}$ & $\begin{array}{l}\text { Depressed skull fracture } \\
\text { Fracture of tibia \& fibula } \\
\text { Fracture ribs } \\
\text { Abrasions- bruises- contused } \\
\text { wounds }\end{array}$ & 27 & Head injury \\
\hline 2 & $\begin{array}{l}\text { Marijuana- } \\
\text { negative driver }\end{array}$ & $\begin{array}{l}\text { Intracerebral hemorrhage } \\
\text { Fracture pelvis, tibia, \& fibula } \\
\text { Fracture ribs } \\
\text { Abrasions- bruises- contused } \\
\& \text { cut wounds }\end{array}$ & 34 & Head injury \\
\hline 3 & $\begin{array}{l}\text { Marijuana- } \\
\text { negative driver }\end{array}$ & $\begin{array}{l}\text { EDH -Black eye } \\
\text { Fracture of tibia (open) } \\
\text { Abrasions- bruises- contused } \\
\text { wound }\end{array}$ & 29 & Head injury \\
\hline 4 & $\begin{array}{l}\text { Marijuana- } \\
\text { negative driver }\end{array}$ & $\begin{array}{l}\text { Depressed skull fracture, } \\
\text { EDH, SDH, Black eye } \\
\text { Abrasions- bruises- contused } \\
\text { wounds }\end{array}$ & 29 & Head injury \\
\hline 5 & $\begin{array}{l}\text { Marijuana- } \\
\text { positive driver }\end{array}$ & $\begin{array}{l}\text { Rupture spleen-intraperitoneal } \\
\text { hemorrhage } \\
\text { Fracture ribs (open) } \\
\text { Abrasions- cut wounds }\end{array}$ & 36 & $\begin{array}{l}\text { Hemorrhage due to } \\
\text { abdominal injury }\end{array}$ \\
\hline 6 & $\begin{array}{l}\text { Marijuana- } \\
\text { positive driver }\end{array}$ & $\begin{array}{l}\text { Depressed skull fracture, } \\
\text { EDH, SDH, Black eye } \\
\text { Fracture ribs (open) } \\
\text { Cut wounds }\end{array}$ & 45 & Head injury \\
\hline
\end{tabular}




\begin{tabular}{|c|c|c|c|c|}
\hline 7 & $\begin{array}{l}\text { Marijuana- } \\
\text { positive driver }\end{array}$ & $\begin{array}{l}\text { Rupture spleen-intraperitoneal } \\
\text { hemorrhage } \\
\text { Fracture ribs \& clavicle } \\
\text { Abrasions- cut wounds }\end{array}$ & 29 & $\begin{array}{l}\text { Hemorrhage due to } \\
\text { abdominal injury }\end{array}$ \\
\hline 8 & $\begin{array}{l}\text { Marijuana- } \\
\text { positive driver }\end{array}$ & $\begin{array}{l}\text { Intraperitoneal hemorrhage } \\
\text { Fracture ribs } \\
\text { Cut wounds }\end{array}$ & 29 & $\begin{array}{l}\text { Hemorrhage due to } \\
\text { abdominal injury }\end{array}$ \\
\hline 9 & $\begin{array}{l}\text { Marijuana- } \\
\text { positive driver }\end{array}$ & $\begin{array}{l}\text { Depressed comminuted skull } \\
\text { fracture, EDH } \\
\text { Fracture radius (open) } \\
\text { Cut wounds }\end{array}$ & 38 & Head injury \\
\hline 10 & $\begin{array}{l}\text { Marijuana- } \\
\text { positive driver }\end{array}$ & $\begin{array}{l}\text { Depressed comminuted } \\
\text { fracture, EDH } \\
\text { Rupture spleen, intraperitoneal } \\
\text { hemorrhage } \\
\text { Fracture pelvis, tibia, \& fibula } \\
\text { Abrasions- bruises- contused } \\
\text { wounds }\end{array}$ & 57 & Head injury \\
\hline 11 & $\begin{array}{l}\text { Marijuana- } \\
\text { positive driver }\end{array}$ & $\begin{array}{l}\text { Depressed comminuted } \\
\text { fracture, EDH } \\
\text { Fracture patella (open) } \\
\text { Abrasions- bruises- contused } \\
\text { wounds }\end{array}$ & 38 & Head injury \\
\hline 12 & $\begin{array}{l}\text { Marijuana- } \\
\text { positive driver }\end{array}$ & $\begin{array}{l}\text { Skull fracture, EDH } \\
\text { Fracture femur } \\
\text { Abrasions-bruises- contused } \\
\text { wounds }\end{array}$ & 38 & Head injury \\
\hline 13 & $\begin{array}{l}\text { Marijuana- } \\
\text { positive driver }\end{array}$ & $\begin{array}{l}\text { Depressed skull fracture, black } \\
\text { eye } \\
\text { Fracture femur } \\
\text { Contused wounds }\end{array}$ & 20 & Head injury \\
\hline 14 & $\begin{array}{l}\text { Marijuana- } \\
\text { positive driver }\end{array}$ & $\begin{array}{l}\text { Rupture spleen, intraperitoneal } \\
\text { hemorrhage } \\
\text { Abrasions - bruises }\end{array}$ & 29 & $\begin{array}{l}\text { Hemorrhage due to } \\
\text { abdominal injury }\end{array}$ \\
\hline 15 & $\begin{array}{l}\text { Marijuana- } \\
\text { positive driver }\end{array}$ & $\begin{array}{l}\text { Skull fracture, EDH } \\
\text { Rupture spleen, intraperitoneal } \\
\text { hemorrhage } \\
\text { Bruises }\end{array}$ & 42 & Head injury \\
\hline 16 & $\begin{array}{l}\text { Marijuana- } \\
\text { positive driver }\end{array}$ & $\begin{array}{l}\text { Depressed comminuted } \\
\text { fracture, EDH } \\
\text { Rupture spleen, intraperitoneal } \\
\text { hemorrhage } \\
\text { Abrasions - bruises }\end{array}$ & 42 & Head injury \\
\hline 17 & $\begin{array}{l}\text { Marijuana- } \\
\text { positive driver }\end{array}$ & $\begin{array}{l}\text { Depressed comminuted } \\
\text { fracture, EDH } \\
\text { Abrasions- bruises- contused } \\
\text { wounds }\end{array}$ & 29 & Head injury \\
\hline
\end{tabular}

EDH: Extradural hemorrhage; SDH: subdural hemorrhage 


\section{DISCUSSION}

Driving under illicit-drug influence is known to have much higher risk of fatal accidents. Cannabis consuming effects on driving are not thoroughly understood and are still controversial (Hostiuc et al., 2018; Das et al., 2020). Such conflicting reports could be due to differences in techniques of consumption, individual tolerance and blood concentrations (Martin et al., 2017). The present study aimed to compare the pattern, severity and clinical outcome of injuries in marijuana smoking drivers, so as to be able to discriminate their clinical course from non-drugged drivers.

In the United States, unexpected rise was reported in the rate of arrests and RTAs in cannabis-positive drivers (Governors Highway Safety Association, 2018). In common with several published studies (Hamed, 2006; Figl et al., 2010; ShahinAbuelfadl, 2015; Martin et al., 2017), results of the current study revealed that 36 drivers $(46.2 \%)$ out of the 78 drivers, who arrived to Tanta University Emergency Hospital for recent RTA and fulfilled eligibility criteria for this study, tested positive for cannabis in urine and 42 drivers $(53.8 \%)$ tested negative.

The high prevalence of cannabispositive drivers involved in crashes could be attributed to reduced drivers' capacity together with performance degradation under cannabis influence which increases the risk of accident. Driving-related cognitive functions and psychomotor skills are considerably reduced, including handeye coordination, attention as well as perception of time and distance (Battistella et al., 2013). Furthermore, it can produce risk-taking behavior that can impair driving skills (Lane et al., 2005; Ramaekers et al., 2006).

Controlled laboratory researches have suggested that, cannabis impairs tasks of attention - whether selective or divided and administrative function (Kelly et al., 2004; Turner, 2007). Experimental studies have declared that cannabis has passive effects on intellectual functions and psychomotor skills. Cannabis affects shortterm memory, ability to process information, maneuverability (tracking) and learning (Ménétrey et al., 2005; Messinis et al., 2006).

In the current study, higher percentage of marijuana-positive drivers belonged to the age group $18-<28$ years, with men outnumbering women. These demographic data come parallel to data gathered by comparable studies in Assiut and Mansoura Governorates (Asaad et al., 2003; Hamed, 2006; A Dawood et al., 2009). They reported more or less similar age and gender prevalence of cannabis abuse in traumatized drivers. Men are reported to be more likely to use almost all types of illicit drugs than women (Center for Behavioral Health Statistics and Quality, 2017). Additionally, Guo et al. (2002) supposed that illicit drug abuse is a youth phenomenon.

In the current study, a significantly higher percentage of marijuana-positive drivers admitted history of drug abuse. Moreover, all drivers in the positive group were smokers. This comes in harmony with data gathered by numerous comparable studies inside and outside Egypt (Ghanem et al., 2000; Hamed, 2006; Figl et al., 2010). The "gateway" pattern of drug initiation could explain such finding according to Bonomo Proimos (2005) and Degenhardt et al. (2009). It supposed that sequence of abused drugs begins with alcohol and tobacco use, followed by cannabis, then other illicit drugs. Furthermore, genetic differences can create a risk of being dependent on single or multiple substances (Sherva et al., 2010).

According to Atkinson et al. (2009), abuse of specific substances might induce individual changes in physiological functioning. This fact might explain the current significant differences in respiratory rate between marijuana-positive drivers and non-drugged drivers. Nevertheless, pulse, blood pressure, temperature, GCS and clinical neurological examination registered no significant differences between 
marijuana-positive drivers and non-drugged drivers.

In the existing study, there was no significant difference in distribution of injuries (total cases among different body parts) between both non-drugged drivers and marijuana smoking drivers. Similar result was obtained by Shahin Abuelfadl (2015) who explained it by occupying an identical seat (drivers' seat) and this led to the same mechanism of injury in both nondrugged and marijuana smoking drivers.

The rate of skull fracture, rupture spleen, intraperitoneal hemorrhage, abrasions and contused wound exhibited significant differences between both nondrugged and marijuana-smoking drivers. Skull fracture was reported in $7.1 \%$ \& $25 \%$ in non-drugged and marijuanasmoking drivers, respectively. In the study done by Shahin Abuelfadl (2015), the incidence of skull fracture was higher $(13.63 \%$ and $43.75 \%$ in non-drugged drivers and marijuana-smoking drivers, respectively). Such higher incidence could be due to variations in seat belt use and air bag-equipped cars between the studies (Martin et al., 2000).

Several authors have investigated cannabis abuse in blood and/or urine of drivers (ComptonBening, 2009; Lacey et al., 2009; Berning et al., 2015). As well, multiple reviews have explored the association between cannabis abuse and driving performance (Lane et al., 2005; Ramaekers et al., 2006; Turner, 2007). Yet, according to available knowledge, none of these studies have discussed injuries' severity in marijuana-smoking drivers. In addition, association between injuries' severity and marijuana level was not established so far.

In the current study, the ISS was non significantly higher and the RTS was non significantly lower in marijuana-smoking drivers compared to non-drugged drivers. The marijuana-positive group showed a significantly higher mortality rate. The ISS and RTS represent anatomical and physiological scoring systems, respectively.
Individuals with short- or long-term use of cannabis may experience changes in physiological functioning that affect the RTS (Atkinson et al., 2009). The significantly higher mortality detected in marijuana-smoking drivers seems reasonable and could be attributed to the higher incidence of fatal injuries (skull fractures, meningeal hemorrhage, rupture spleen and intraperitoneal hemorrhage) among them.

In the present study, a significant positive strong correlation could be observed between THC level and ISS, together with significant negative strong correlation between THC levels and RTS. Additionally, among marijuana-positive drivers, non-survivors had significantly higher median THC level than that of survivors. This might direct attention toward a dose-dependent effect of marijuana. Such effect has been demonstrated in certain aspects of vehicle control, including maintaining a distance from the vehicle in-front, steering, reaction time, and lane-keeping (Martin et al., 2017). However, these findings disagree with those obtained by Shahin Abuelfadl (2015) who found no significant correlation of THC level with ISS or RTS as well as no significant difference in THC level between survivors and non-survivors.

In the present study, four non-drugged drivers possibly died due to severe head injury (their ISS was 27, 34, 29, 29). In marijuana smoking drivers, hemorrhage due to abdominal injury was the possible cause of death in four drivers (their ISS was 29, 36, 29, 29). Meanwhile, head injury with different spectrum of skull fractures and meningeal hemorrhage - was the possible cause of death in the remaining nine drivers (ISS $=29,42,42,38,38,38$, $57,45,20)$.

Analyzing drivers' urine samples is considered a major limitation of this study, as THC is excreted and exists in urine only as inactive metabolite. Hence, positive testing indicates cannabis use, but does not confirm recent intake and the time of intake 
cannot be inferred (Ramaekers et al., 2009; Sewell et al., 2009). Including blood samples that detect active THC metabolites, in further upcoming studies, might reveal a more truthful impression. Another limitation was uncalculated sample size. Therefore, future comparable studies with registration of road traffic injury victims in several emergency hospitals countrywide are mandatory and will yield more precise and illustrative data that can help in reduction of morbidity and mortality among those victims.

\section{CONCLUSIONS}

From the results of the present study, it could be concluded that driving under the effect of marijuana is a public health problem that affects the younger and productive age groups. Marijuana smoking increases the mortality rate in victims of RTAs. There is a strong correlation between THC level and both ISS and RTS. In addition, there is a dose-dependent effect of marijuana smoking (THC level is higher among the non-surviving marijuanasmoking drivers).

\section{RECOMMENDATIONS}

1- Driving under the effect of marijuana should be legally prohibited to reduce the incidence of RTAs and the mortality rate related to them.

2- Periodic checks for the presence of marijuana and other drugs of abuse should be done.

3- Victims of RTAs should be routinely checked for marijuana and other drugs of abuse in urine to help in identification of the magnitude of this problem to design and implement effective solutions.

4- Further studies with larger sample size and with detection of THC level in blood are recommended to confirm or refute the suggested effect of marijuana on driving and RTAs.

\section{REFERENCES}

A Dawood, A.E.-W.; Shehata, M.M.; Abdel-Hady, R.H.; Abdel-Aal, K.M. and Yassa, H.A. (2009): Risk Factors Lead to Bango Abuse Among Drivers and Secondery School Students in Assiut Province. Iranian Journal of Toxicology, 2(4): 246- 253.

Andrews, R.; Murphy, K.G.; Nahar, L. and Paterson, S. (2015): Cannabinoid concentrations detected in fatal road traffic collision victims compared with a population of other postmortem cases. Clinical Chemistry, 61: 12561264.

Arterberry, B.J.; Treloar, H.R.; Smith, A.E.; Martens, M.P.; Pedersen, S.L. and Mccarthy, D.M. (2013): Marijuana use, driving, and related cognitions. Psychology of Addictive Behaviors, 27(3): 854- 860

Asaad, T.a.A.; Okasha, T.A.; El-Khouly, G.A. and Azim, K.a.A. (2003): Substance abuse in a sample of Egyptian schizophrenic patients. Addictive Disorders \& Their Treatment, 2(4): 147- 150.

Atkinson, A.; Anderson, Z. and Hughes, K. (2009): Interpersonal violence and illicit drugs. Centre for Public Health. Liverpool, John Moores University. https://www.who.int/violencepreventi on/interpersonal_violence and illicit drug use.pdf

Battistella, G.; Fornari, E.; Thomas, A.; Mall, J.-F.; Chtioui, H.; Appenzeller, M., et al. (2013): Weed or wheel! FMRI, behavioural, and toxicological investigations of how cannabis smoking affects skills necessary for driving. PloS One, 8(1): e52545.

Bergeron, J. and Paquette, M. (2014): Relationships between frequency of driving under the influence of cannabis, self-reported reckless driving and risk-taking behavior observed in a driving simulator. Journal of Safety Research, 49: 19-24.

Berning, A.; Compton, R. and Wochinger, K. (2015): Results of the 
2013-2014 national roadside survey of alcohol and drug use by drivers. Journal of Drug Addiction, Education, and Eradication, 11(1): 47.

Bonomo, Y. and Proimos, J. (2005): Substance misuse: alcohol, tobacco, inhalants, and other drugs. BMJ, 330(7494): 777- 780.

Center for Behavioral Health Statistics and Quality (2017): Results from the 2016 National Survey on Drug Use and Health: Detailed Tables. Rockville, Maryland, Substance Abuse and Mental Health Services Administration.

Compton, R. and Bening, A. (2009): Results of the 2007 National Roadside Survey of Alcohol and Drug Use by Drivers. In Traffic Safety Facts. Washington, DC: National Highway and Traffic Safety Administration. Office of Behavioral Safety Research. DOT HS 811175

Das, S.; Tran, L.-N. and Theel, M. (2020): Understanding patterns in Marijuana impaired traffic crashes. Journal of Substance Use, 1-9.

Degenhardt, L.; Chiu, W.; Conway, K.; Dierker, L.; Glantz, M.; Kalaydjian, A., et al. (2009): Does the 'gateway'matter? Associations between the order of drug use initiation and the development of drug dependence in the National Comorbidity Study Replication. Psychological Medicine, 39(1): 157167.

Drummer, O.H.; Kourtis, I.; Beyer, J.; Tayler, P.; Boorman, M. and Gerostamoulos, D. (2012): The prevalence of drugs in injured drivers. Forensic Science International, 215(13): 14-17.

Figl, M.; Pelinka, L.E.; Weninger, P.; Walchetseder, C.; Mauritz, W.; Hertz, H., et al. (2010): Urine toxicology screening in Austrian trauma patients: a prospective study. Archives of Orthopaedic and Trauma Surgery, 130(7): 883- 887.
Ghanem, A.A.; Farid, M. and Nasr, M.E. (2000): Psychoactive substance use among trauma victims: A preliminary study. J. Legal Med. Forensic Sci. , 12(4): 289- 298.

Governors Highway Safety Association (GHSA) (2018): Traffic safety impacts of Marijuana legalization [Online]. Available:

https://www.ghsa.org/resources/Marij uanaImpacts 18.

Guo, J.; Hill, K.G.; Hawkins, J.D.; Catalano, R.F. and Abbott, R.D. (2002): A developmental analysis of sociodemographic, family, and peer effects on adolescent illicit drug initiation. Journal of the American Academy of Child \& Adolescent Psychiatry, 41(7): 838- 845.

Hamed, R.H.A. (2006): Toxicological screening of trauma patients in urban and rural areas. M.D. Thesis, Mansoura University.

Hartman, R.L. and Huestis, M.A. (2013): Cannabis effects on driving skills. Clinical Chemistry, 59: 478-492.

Hostiuc, S.; Moldoveanu, A.; Negoi, I. and Drima, E. (2018): The association of unfavorable traffic events and cannabis usage: a metaanalysis. Frontiers in Pharmacology, 9 doi: 10.3389/fphar.2018.00099

James, S.L.; Lucchesi, L.R.; Bisignano, C.; Castle, C.D.; Dingels, Z.V.; Fox, J.T., et al. (2020): Morbidity and mortality from road injuries: results from the Global Burden of Disease Study 2017. Injury Prevention. doi:10.1136/injuryprev-2019-043302.

Joseph, S. and Ronald, C.B. (2006):

Focus on urine drug monitoring: Are your patients compliant, diverting, or supplementing the drugs that are prescribed for their chronic pain? Practical Pain Management, 6(2): 6067

Kelly, E.; Darke, S. and Ross, J. (2004): A review of drug use and driving: epidemiology, impairment, risk factors and risk perceptions. Drug and Alcohol 
Review, 23(3): 319-44 .

Kumaraguru, J.; Isaac, A.; Kandasamy, N.; Meacock, L.; Strom, L.; Luck, S., et al. (2015): Clinical applications of the Injury Severity Scoring system (ISS): The clinical, radiological, educational, research and economic implications on reporting in trauma. Results from a tertiary trauma centre in UK. 2015. European Congress of Radiology-ECR.

Lacey, J.H.; Kelley-Baker, T.; FurrHolden, D.; Voas, R.B.; Romano, E.; Ramirez, A., et al. (2009): 2007 national roadside survey of alcohol and drug use by drivers: drug results. No. DOT HS 811249 United States. National Highway Traffic Safety Administration

Lane, S.D.; Cherek, D.R.; Tcheremissine, O.V.; Lieving, L.M. and Pietras, C.J. (2005): Acute marijuana effects on human risk taking. Neuropsychopharmacology, $30(4)$ : 800-809.

Martin, J.-L.; Gadegbeku, B.; Wu, D.; Viallon, V. and Laumon, B. (2017): Cannabis, alcohol and fatal road accidents. PLoS One, 12(11): e0187320. doi: 10.1371/journal.pone.0187320

Martin, P.G.; Crandall, J.R. and Pilkey, W.D. (2000): Injury trends of passenger car drivers in frontal crashes in the USA. Accident Analysis \& Prevention, 32(4): 541- 557.

Ménétrey, A.; Augsburger, M.; Favrat, B.; Pin, M.A.; Rothuizen, L.E.; Appenzeller, M., et al. (2005): Assessment of driving capability through the use of clinical and psychomotor tests in relation to blood cannabinoids levels following oral administration of $20 \mathrm{mg}$ dronabinol or of a cannabis decoction made with 20 or $60 \mathrm{mg} \quad \Delta 9$-THC. Journal of Analytical Toxicology, 29(5): 327-338.

Messinis, L.; Kyprianidou, A.; Malefaki, S. and Papathanasopoulos, P. (2006): Neuropsychological deficits in long-term frequent cannabis users. Neurology, 66(5): 737- 739.

Mir, M.U.; Khan, I.; Ahmed, B. and Razzak, J.A. (2012): Alcohol and marijuana use while driving--an unexpected crash risk in Pakistani commercial drivers: a cross-sectional survey. BMC Public Health, 12(1): 145.

Nayeem, N.; Barltrop, A. and Kotecha, M.B. (1992): Care of road traffic accident victims in a district general hospital. Annals of the Royal College of Surgeons of England, 74(3):212217

Obada, S. (2009): Traffic Problems in Egypt and Emirates. Comparative study. Police Academy, Cairo, 12-19.

Petridou, E. and Antonopoulos, C. (2017): Injury epidemiology. In: Quah, S.R. and Cockerham, W.C. (eds.) International Encyclopedia of Public Health $2^{\text {nd }}$ ed. Oxford, Elsevier; 258274

Ramaekers, J.G.; Berghaus, G.; Van Laar, M. and Drummer, O.H. (2009): Dose related risk of motor vehicle crashes after cannabis use: an update. In: Verster, J.C.; PandiPerumal, S.R.; Ramaekers, J.G.; de Gier, J.J. (eds) Drugs, driving and traffic safety Birkhauser, Basel, Switzerland, Springer; 477- 499.

Ramaekers, J.G.; Kauert, G.; Van Ruitenbeek, P.; Theunissen, E.L.; Schneider, E. and Moeller, M.R. (2006): High-potency marijuana impairs executive function and inhibitory motor control. Neuropsychopharmacology, 31: 22962303.

Sewell, R.A.; Poling, J. and Sofuoglu, M. (2009): The effect of cannabis compared with alcohol on driving. American Journal on Addictions, 18(3): 185-93.

Shahin, M. and Abuelfadl, A. (2015): Toxicological and Forensic Evaluation of Injury Pattern and Mortality in Marijuana Smoking Drivers and Non- 
Drugged Drivers: a Comparative Study. Ain Shams Journal of Forensic Medicine and Clinical Toxicology, 25(2): 93-103.

Sherva, R.; Kranzler, H.R.; Yu, Y.; Logue, M.W.; Poling, J.; Arias, A.J., et al. (2010): Variation in nicotinic acetylcholine receptor genes is associated with multiple substance dependence phenotypes.

Neuropsychopharmacology, 35(9): 1921- 1931.

Turner, B.M.A. (2007): Sex, drugs, and driving: The effects of marijuana. Dissertation, University of Iowa

World Health Organization (WHO) (2018): Global Status Report on Road Safety 2018. Geneva, WHO; 1- 424 


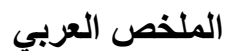 \\ في حوادث المرور على الطرق، هل يتأثر نمطوشُدة ونتائج إصابات السائقين بتعاطي

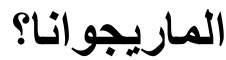

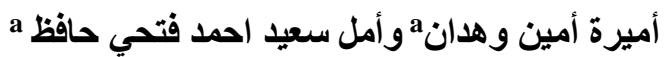

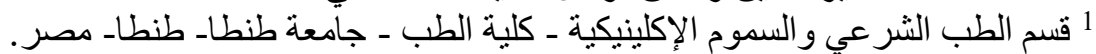

مقدمة: تمثل حو ادث الطرق المرورية مساهماً رئيسياً في العبء العالمي للأمر اض. وقد وقد وجد أن

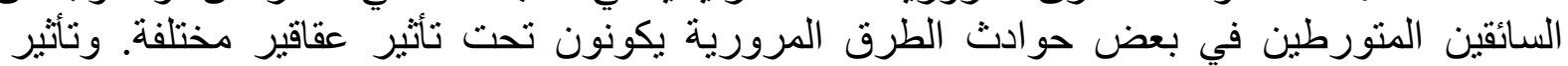

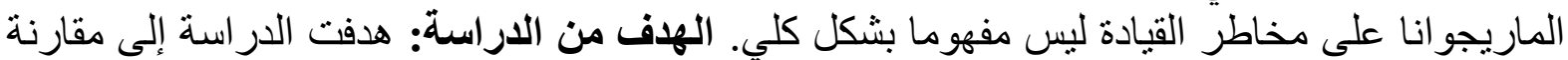

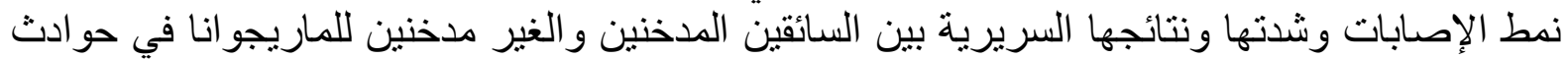

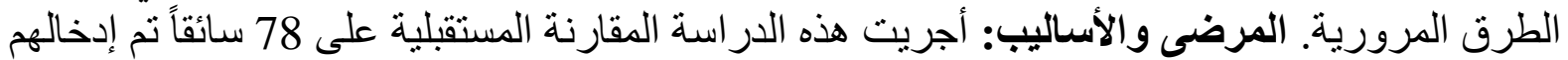

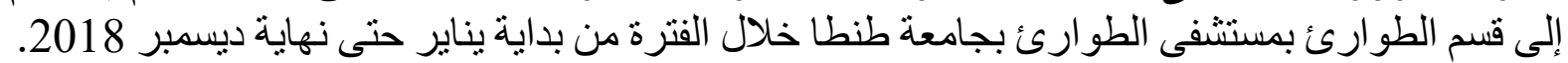

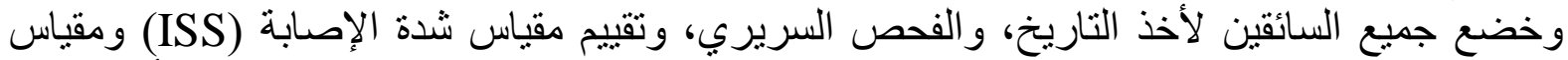

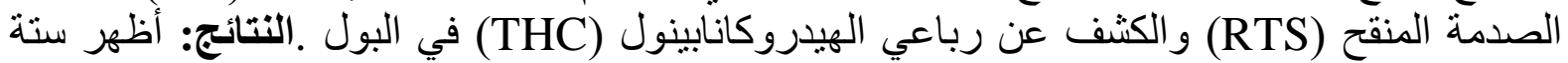

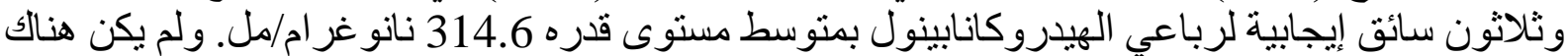

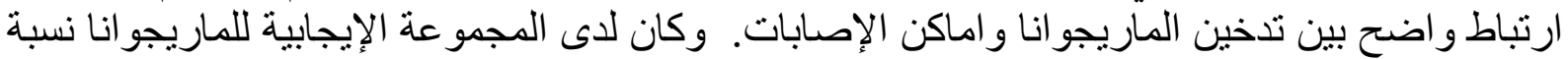

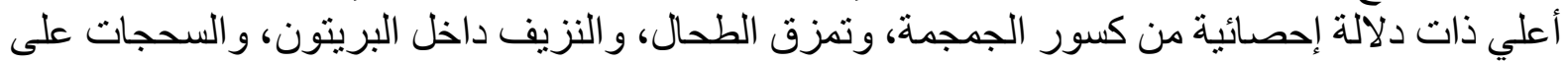

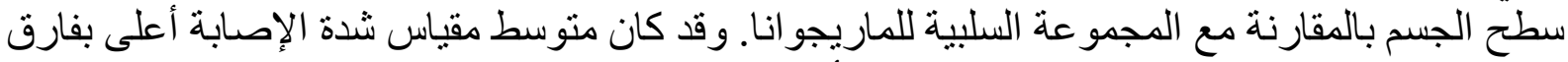

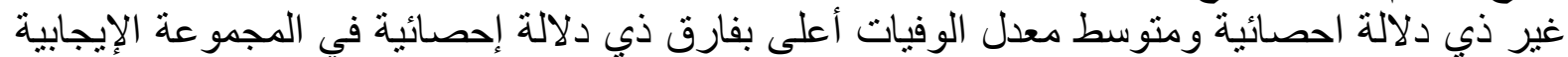

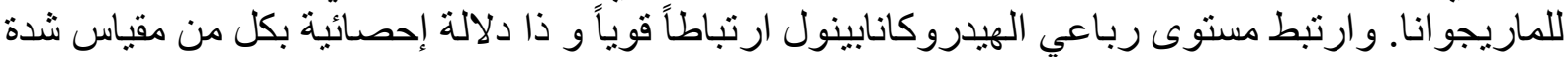

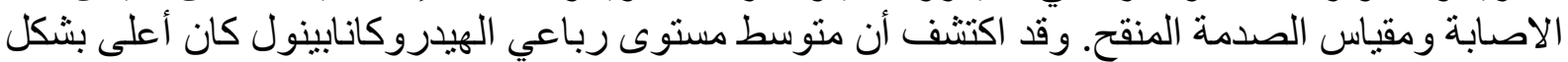

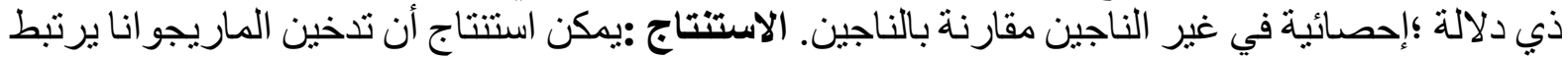

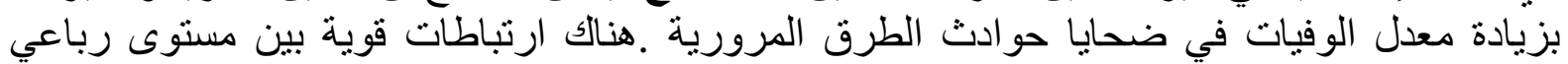

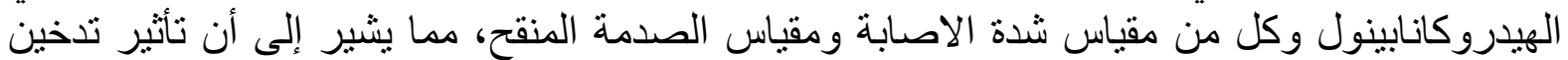
الماريجو انا يعتمد على الجر عة مغن. 